\title{
BMJ Open Effectiveness and optimal dosage of resistance training for chronic non- specific neck pain: a protocol for a systematic review with a qualitative synthesis and meta-analysis
}

Jonathan Price, ${ }^{\circledR 1}$ Alison Rushton, ${ }^{\circledR 2}$ Isaak Tyros, ${ }^{3}$ Nicola R Heneghan ${ }^{\odot} 2$

To cite: Price J, Rushton A, Tyros I, et al. Effectiveness and optimal dosage of resistance training for chronic nonspecific neck pain: a protocol for a systematic review with a qualitative synthesis and meta-analysis. BMJ Open 2019;9:e025158. doi:10.1136/ bmjopen-2018-025158

- Prepublication history and additional material for this paper are available online. To view these files, please visit the journal online (http://dx.doi. org/10.1136/bmjopen-2018025158).

Received 4 July 2018

Revised 4 December 2018 Accepted 20 December 2018

Check for updates

(C) Author(s) (or their employer(s)) 2019. Re-use permitted under CC BY-NC. No commercial re-use. See rights and permissions. Published by BMJ.

For numbered affiliations see end of article.

Correspondence to Dr Nicola R Heneghan; n.heneghan@bham.ac.uk

\section{ABSTRACT}

Introduction The prevalence of neck pain is increasing rapidly with a high percentage of patients going on to experience recurrent or chronic symptoms. The resulting pain and disability are commonly managed using a variety of treatments including exercise. Resistance training exercise aimed at the neck and shoulders is advocated to treat chronic non-specific neck pain (CNSNP), however the dosage of prescribed exercise varies considerably between studies. The aim of this study is to evaluate the effectiveness of resistance training in CNSNP and to determine an optimal dosage that should be prescribed in clinical practice.

Methods and analysis A systematic review with qualitative synthesis and meta-analysis will be conducted according to the Preferred Reporting Items for Systematic Reviews and Meta-Analyses Protocols. Cumulative Index to Nursing and Allied Health Literature, Excerpta Medica Database, Medical Literature Analysis and Retrieval System Online, PEDro, Zetoc, Index to Chiropractic Literature ChiroAcces, PubMed, grey literature sources and key journals will be searched. Randomised clinical trials investigating resistance training exercise in adults with CNSNP using outcome measures of pain and/or disability will be eligible for inclusion. Two reviewers will independently screen for eligibility, extract data and assess risk of bias (Cochrane risk of bias tool) with a third reviewer mediating in cases of disagreement. Data will be synthesised qualitatively to investigate intervention effectiveness and to determine the effect of exercise dosage on pain and disability. Meta-analysis using a random-effects model will be conducted where sufficient clinical homogeneity exists. The strength of the overall body of evidence will be assessed and reported using Grading of Recommendations Assessment, Development and Evaluation.

Ethics and dissemination This study raises no ethical issues. Results will inform exercise prescription to improve management of CNSNP. Results will be published in a peer-reviewed journal and presented at conferences.

PROSPERO registration number CRD42018096187.
Strengths and limitations of this study

- The protocol is written in accordance with the Preferred Reporting Items for Systematic Reviews and Meta-Analyses Protocols guidelines.

- This protocol states an a priori method of both qualitative and quantitative synthesis.

- The overall quality of evidence will be reported using Grading of Recommendations Assessment, Development and Evaluation.

- First evidence synthesis investigating the effect of exercise dosage on chronic non-specific neck pain.

- The criteria used to determine intervention eligibility are broad which will result in a high degree of intervention heterogeneity reducing confidence in results.

\section{BACKGROUND}

Neck pain is experienced by $50 \%$ of the population annually ${ }^{2}$ and is now more common than low back pain among officebased workers. ${ }^{3}$ Neck pain disability is considerable and is now ranked fourth among global disabilities, ahead of anxiety disorders, diabetes and osteoarthritis. ${ }^{4}$ The cause of neck pain, however, is not well- understood as the attributable pathoanatomical cause is often absent. ${ }^{5}$ The prognosis of the first onset of neck pain is poor, with pain and disability often recurring $^{6-9}$ and lasting for $1-5$ years in $50 \%-70 \%$ of individuals. ${ }^{18}$ Experiencing neck pain symptoms for $>3$ months without a specific pathoanatomical cause is described as chronic non-specific neck pain (CNSNP).

Multiple national and best practice guidelines suggest that CNSNP is best managed using a multimodal approach including a combination of education, advice, manual therapy and exercise interventions. ${ }^{5} 1011$ Although a variety of exercises is used in clinical practice, a Cochrane review investigating 
different exercise interventions provides moderate levels of evidence in support of strengthening and/or endurance exercise. ${ }^{12}$ Further to this, a recent systematic review and meta-analysis that performed a more focused review of work place interventions in a specific population of office workers also demonstrated that strengthening and/or endurance exercise performed in the work place has the most beneficial effect on pain and disability when compared with other interventions. ${ }^{13}$ The strengthening and endurance programmes reported could also be described as resistance training.

Resistance training refers to any exercise used to increase muscle strength, endurance, power, hypertrophy or motor performance by an individual applying a force against resistance. ${ }^{14}$ Use of specific sets, repetitions, load and frequency (components of exercise dosage) based on a defined protocol ${ }^{15-17}$ differentiates strengthening from endurance training. Despite this, the description of exercises within the neck-pain literature is confusing. For example, the same exercise protocol using the same dosage has been described as "endurance training, ${ }^{\text {'8 }}$ craniocervical flexion retraining, ${ }^{19}$ and 'strengthening, ${ }^{20}$ In addition to inconsistent terminology, the Cochrane review identified that the dosage of exercise used for 'strengthening' exercises varied considerably across studies. ${ }^{21-23}$ The inconsistency in exercise dosage provides little guidance as to the optimal dosage of resistance training exercises to be prescribed in a clinical setting.

Other authors have used consistent terminology and protocols to describe strengthening or endurance exercises. ${ }^{21-24}$ However, in these studies, the exercise dosage used is designed to change a performance outcome in a healthy population (ie, strength or endurance) and not designed to decrease pain or disability. Exercises based on protocols intended for a performance outcome in a healthy population have limited external validity for a patient population where the aim is to reduce pain and disability, thus contributing to a lack of clarity of optimal dosage of resistance training exercises that should be used in a patient population. Although the Cochrane review highlighted that research could investigate optimal dosage by comparing similar types of exercises, ${ }^{12}$ they excluded studies using exercise as a comparator ${ }^{29-31}$ and were, therefore, unable to investigate the effect of exercise dosage on pain and/or disability. There is now a call from experts ${ }^{32-34}$ and professional bodies ${ }^{35}$ for future research to establish the optimal dosage of exercise to decrease pain or disability in patient populations. There has been no systematic review to date that has focused specifically on resistance training to evaluate its effectiveness or determine optimal dosage in CNSNP.

\section{Aim}

The primary aim is to evaluate the effectiveness of resistance training aimed at the neck and shoulders on pain and/or disability of CNSNP patients. A secondary aim is to investigate whether changing the dosage of specific resistance training protocols influences their effectiveness on pain and/or disability.

\section{METHODS \\ Protocol and registration}

The protocol is reported in line with the Preferred Reporting Items for Systematic Review and Meta-Analyses Protocols ${ }^{36}$ (online supplementary file 1 ).

\section{Eligibility criteria}

Studies will be selected for review should they meet the following criteria:

\section{Population}

Studies will be included if the population comprises adults aged 18-70 experiencing non-specific neck pain for $\geq 3$ months. Specific pathology such as cervicogenic headache, cervicogenic dizziness, whiplash associated disorder, cervical radiculopathy, fractures, spondylolisthesis, inflammatory disease, fibromyalgia, cancer, neck pain resulting from surgery, post-traumatic stress disorder or cervicobrachial syndrome will be excluded.

\section{Intervention}

Eligible studies will describe an exercise programme consisting of exercises targeted at the neck or shoulders where an individual applies a force against resistance (eg, dumbbell, resistance band, gravity). Programmes that also include other types of exercises such as stretching or aerobic exercises will only be permitted if used as part of a warm up or cool down. Any exercise programme that includes a variety of exercise types (resistance training, stretching, aerobic exercise) and where it is not clear that the focus is on resistance training will be excluded. Any exercise therapy given as part of a multidisciplinary treatment or exercise that requires manual therapy techniques by a trained individual will also be excluded.

\section{Comparison}

Any comparison will be permitted, including with another exercise group, placebo, other therapies or usual care. Exercise programmes plus another intervention (eg, manual therapy, education etc) will be included provided a comparison group includes the same intervention.

\section{Outcome measures}

Studies will be included if they used patient reported measures of pain (eg, Visual Analogue Scale (VAS), Numeric Pain Rating Scale or functional disability (eg, Neck Disability Index (NDI), Neck Pain and Disability Scale).

\section{Study design}

Randomised, controlled, clinical trials only will be permitted. Pilot or feasibility studies will be excluded. 


\section{Other}

Studies not written in English will be excluded at full text and will be reported within the Preferred Reporting Items for Systematic Reviews and Meta-Analyses (PRISMA) flow diagram. There are no limitations on publication date.

\section{Patient involvement}

The study was conceived from our clinical working with patients with spinal complaints over many years and their views were used to inform aspects of the methods of the review protocol. Patient users will be invited to contribute to stages of synthesis and interpretation of findings to inform conclusions. Study findings will be disseminated to patients via conference presentations, including the Centre of Precision Rehabilitation for Spinal Pain, Patient and Public Involvement Group.

\section{Information sources}

The search will employ sensitive topic-based strategies designed for each database from inception to 30 November 2018. Searches will be completed using the National Institute for Health and Care Excellence evidence database website (https://hdas.nice.org.uk/) and will include the following databases: Cumulative Index to Nursing and Allied Health Literature (CINAHL), Excerpta Medica Database, Medical Literature Analysis and Retrieval System Online and PubMed. PEDro and Index to Chiropractic Literature will be searched separately. Selected internet sites and indices will be used, including Turning Research into Practice. Manual search of key journals for relevant articles, articles in press, or published ahead of print will include Spine, European Spine Journal, Journal of Orthopaedic and Sports Physical Therapy, Strength and Conditioning Journal and The Journal of Strength and Conditioning. Publishers such as Elsevier, Wiley and Springer will also be manually searched for articles. Grey literature search $^{37}$ will include Zetoc and OpenGrey. Reference lists of all included trials will be reviewed for any relevant articles.

\section{Search strategy}

Initial scoping searches have refined the search terms and inclusion criteria to account for the inconsistent terminology and a range of of exercises described within the literature. The search terms have been kept broad to ensure a sensitive search strategy. Terms and keywords are presented in table 1 using Population Intervention Comparison Outcome Study Design. ${ }^{38} \mathrm{JP}$, subject matter expert, will conduct the electronic database searches by adapting to the unique search features of each individual database. See table 2 for an example of search strategy for CINAHL. As resistance training will be compared with any other treatment, including other exercises, no search terms will be used for comparator interventions.

\section{Study records}

Data management

All search results will be imported into Endnote ${ }^{39}$ software for managing bibliographies.

\section{Selection process}

JP and IT (subject matter experts) will independently screen the titles and abstracts for inclusion. Eligibility criterion will be graded as eligible/not eligible/might be eligible. A study will be considered 'might be eligible' when it cannot be excluded on the basis of its title and abstract. Full copies of all potentially eligible papers will be independently screened by two reviewers (JP and IT). A third reviewer (NH) (subject matter and methodology expert) will mediate in the event of disagreement following discussion. The study selection process will be summarised using a PRISMA flow diagram. ${ }^{40}$ A kappa statistic will be used to assess agreement at both title/abstract and full-text screening stages between the two reviewers, where $0.40-0.59$ is fair agreement, $0.60-0.74$ is good agreement and 0.75 or more is excellent agreement. $^{3741}$

\section{Data collection and extraction}

Data will be extracted independently by JP and IT using the Cochrane 'Data collection forms for intervention

Table 1 Search terms

\begin{tabular}{|c|c|c|c|}
\hline Population & Comparison & Outcome & Study design \\
\hline Chronic neck pain & Strengthening & Disability & Controlled clinical trial \\
\hline Cervicalgia & Strength endurance & Numeric Rating Scale & \\
\hline Cervicodynia & Strength training & Visual Analogue Scale & \\
\hline Trapezius myalgia & Cranio-cervical flexion & Neck Pain and Disability Scale & \\
\hline Neck symptoms & Exercise & & \\
\hline \multirow[t]{2}{*}{ Neck sprain } & Exercise training & & \\
\hline & Exercise programme & & \\
\hline
\end{tabular}


Table 2 Search strategy to be used for the Cumulative Index to Nursing and Allied Health Literature electronic database (CINAHL)

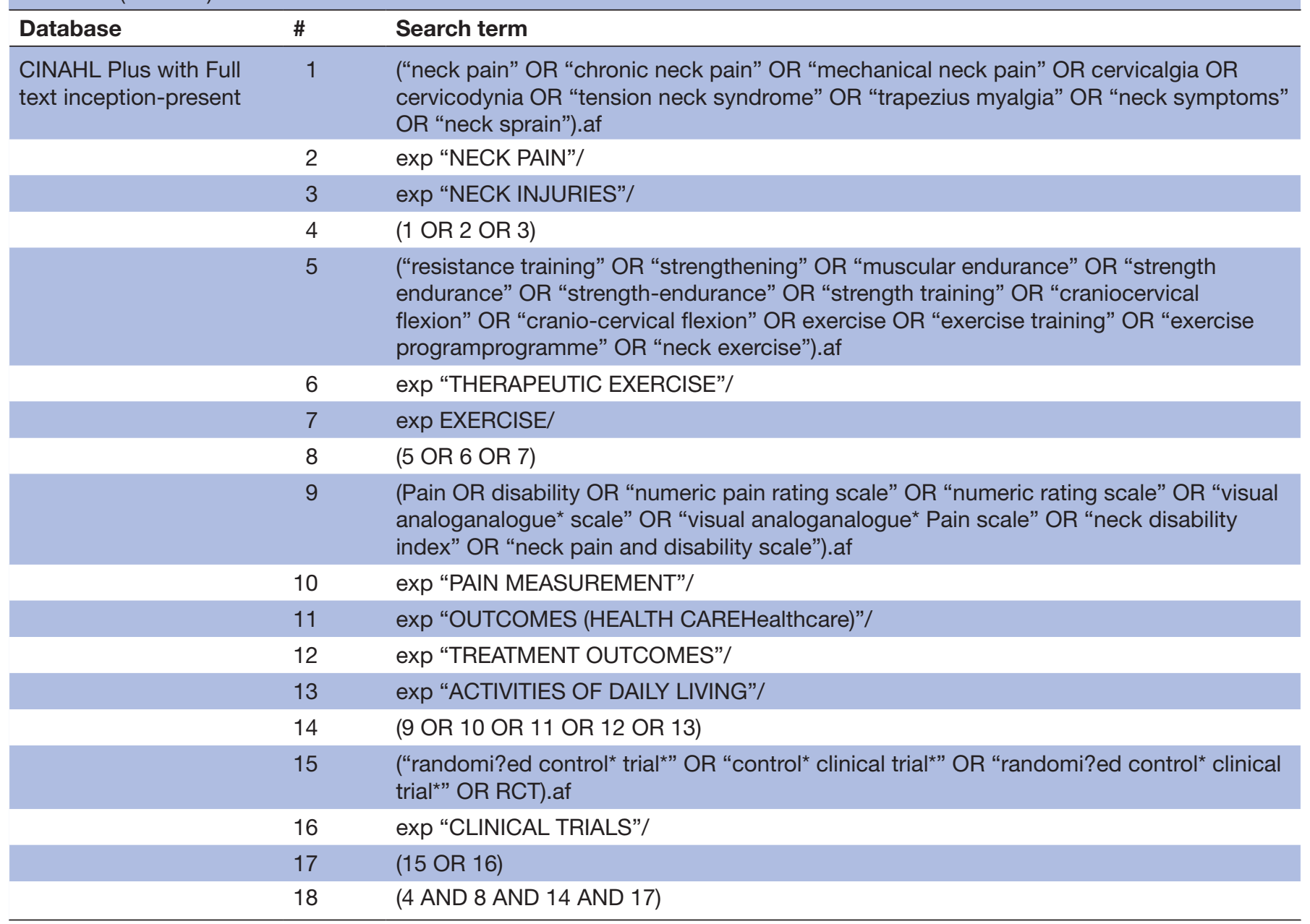

reviews: Randomised Controlled Trial (RCTs) and non-RCTs' ${ }^{42}$ The form will be piloted and edited prior to data extraction on five randomly selected studies. A third reviewer $(\mathrm{NH})$ will mediate in the event of disagreement following discussion. The following data will be extracted: trial author, publication date, participant information (gender, age), sample size, study setting, intervention data, power calculations, outcome measures, follow-up period and main results. The outcomes of interest will be any continuous or dichotomous measure of pain and/or disability only and measured with, for example, VAS, Numeric Rating Scale or NDI. Outcome data will not be converted during data extraction and will be extracted as originally reported in each study. Where there are multiple reports of the same study, these will be reported on the same data extraction form. Detailed intervention data will be extracted using the 12-item TIDier Checklist. ${ }^{43}$ Although designed to aid in reporting of interventions, using this checklist to extract intervention data will allow an assessment of intervention fidelity and clinical heterogeneity among different studies. Due to the inconsistent terminology used within the literature to describe exercise interventions as either strengthening or endurance we will use a classification system developed by experts to describe exercise interventions in TIDier. ${ }^{44}$

\section{Dealing with missing data}

Researchers will be contacted for additional data when data are missing or ambiguous. If missing summary data cannot be obtained, an imputation method will be used to estimate the mean and variance values. ${ }^{45}$

\section{Assessment of risk of bias of included studies}

Internal validity of the included trials will be assessed independently by two reviewers (JP, IT) using the Cochrane Risk of Bias tool. ${ }^{37}$ A third reviewer (NH) will mediate in situations of disagreement. Each domain will be judged as 'unclear', 'low' or 'high' risk of bias. ${ }^{37}$ Cohen's $k$ will be used to assess agreement between the two reviewers. ${ }^{37}$

\section{Assessment of heterogeneity}

Clinical heterogeneity will first be assessed using the 'Characteristics of Included Studies' table to compare sample characteristics, outcome measures and brief intervention data. ${ }^{46}$ As scoping searches have identified a large degree of variability in exercise interventions 
reported within the literature, we will use the data collected from the TIDier Checklist to explore intervention heterogeneity. Interventions will be assessed for heterogeneity between studies with respect to the classification of exercises used, dosage of exercises and how they were delivered (group class, one to one, home exercise programme). If significant clinical heterogeneity exists, statistical heterogeneity will not be assessed and a qualitative synthesis of data will be performed. Should clinical homogeneity exist between studies, statistical heterogeneity will be assessed using the $\mathrm{I}^{2}$ statistic. Interpretation of the $\mathrm{I}^{2}$ will be based on the Cochrane Handbook for Systematic Review of Interventions, where $0 \%-40 \%$ is low, $30 \%-60 \%$ is moderate, $50 \%-90 \%$ is substantial and $75 \%-100 \%$ is considerable heterogeneity. ${ }^{47}$ Groups of studies in which heterogeneity is low $\left(\mathrm{I}^{2}<50 \%\right)$ will be assessed using meta-analysis. ${ }^{47}$ The $p$ value from the $\chi^{2}$ test will also be considered using a $p$ value $<0.10$ to determine significant heterogeneity. Groups of studies which are clinically and statistically homogenous will be quantitatively synthesised using meta-analysis. ${ }^{37}$

\section{Data synthesis}

The process of searching and numbering of papers included/excluded will be reported on a PRIMSA flow chart. From a scoping search it is anticipated that clinical heterogeneity will exist with respect to intervention. Therefore, a qualitative synthesis will summarise the evidence using previously documented guidance and stages. ${ }^{48} 49$

\section{Qualitative synthesis}

\section{Developing a preliminary synthesis of findings of included studies}

The findings of the included studies will be synthesised by tabulating study characteristics, results and risk of bias. Detailed intervention data will be tabulated using the TIDier Checklist. ${ }^{43}$ The effect of resistance training on pain and disability will be reported separately using textual descriptions.

\section{Exploring relationships within and between trials}

The relationship between studies will be reported visually using forest plots without a pooled estimate. A potential moderator variable table will be used to present study results (mean difference plus 95\% CIs) and exercise dosage information to investigate the effect of dosage on outcomes for studies that use the same resistance training protocols. ${ }^{48}$ A textual description of exercise dosage will also be completed.

\section{Assessing the robustness of the synthesis}

Potential biases in the review process will be reported in the 'Discussion' section of the final published paper. The quality of the evidence will be assessed using Grading of Recommendations, Assessment, Development and Evaluation. ${ }^{3750}$ Quality will be rated as 'high' 'moderate' 'low' or 'very low'. High quality evidence would indicate that further research is unlikely to change our confidence in the findings. Low quality evidence would suggest further research would likely have an important impact on findings, and very low quality evidence would indicate significant uncertainty of any current effect. ${ }^{3750}$

\section{Quantitative synthesis}

Meta-analysis using a random effects model will be conducted to quantitatively synthesise data if studies are clinically homogenous and statistical heterogeneity is low. ${ }^{37}$ Continuous outcomes will be analysed using either mean difference or standardised mean difference (with 95\% CIs), if different measurement scales are used. Dichotomous outcomes will be analysed using risk ratios with 95\% CIs. Data will be represented quantitatively using tables and graphically using a forest plot. Pooled-intervention effect estimates will be calculated using a weighted average of the intervention effects estimated in the individual studies. ${ }^{37}$

\section{Sensitivity analysis}

A sensitivity analysis will be conducted by investigating whether the following influence meta-analysis:

- Risk of bias (omitting studies that were judged as high risk of bias).

- Missing data (omitting studies that were missing mean and variance values).

\section{Meta-bias}

To avoid publication bias, grey literature will be searched. Publication bias will be assessed graphically using funnel plots should a minimum of 10 studies be included in a meta-analysis. ${ }^{51}$ Outcome bias will be assessed using the Outcome Reporting Bias in Trials classification system. ${ }^{52}$

\section{Amendments}

Any required amendments to this protocol will be reported with date, description of amendment, and rationale for amendment and reported in the final publication and PROSPERO.

\section{Ethics and dissemination}

This study raises no ethical issues. The results of this systematic review and meta-analysis will be published in a peer-reviewed journal and presented at national and international conferences.

\section{Author affiliations}

${ }^{1}$ Musculoskeletal Physiotherapy Services, Birmingham Community Healthcare NHS Foundation Trust, Birmingham, UK

${ }^{2}$ Centre of Precision Rehabilitation for Spinal Pain (CPR Spine), School of Sport, Exercise and Rehabilitation Sciences, College of Life and Environmental Sciences, University of Birmingham, Birmingham, UK

${ }^{3}$ Edgbaston Physiotherapy Clinic, Birmingham, UK

Contributors $\mathrm{JP}$ is the $\mathrm{Cl}$ leading the protocol development, analyses, and dissemination. NH and AR are supervisors for the study, ensuring rigour and quality. JP and IT are the first and second reviewers. NH is the third reviewer. All authors (JP, IT, NH, AR) have contributed to the design and development of the protocol and have contributed to manuscript draft. All authors have read, provided feedback and approved the final manuscript. 
Funding This systematic review will be completed as part of the Heath Education England funded Clinical Academic Internship Programme. Academic support provided from University of Birmingham and the Centre of Precision Rehabilitation for Spinal Pain including patient users involved in the interpretation of the results.

Competing interests None declared.

Patient consent for publication Not required.

Provenance and peer review Not commissioned; externally peer reviewed.

Open access This is an open access article distributed in accordance with the Creative Commons Attribution Non Commercial (CC BY-NC 4.0) license, which permits others to distribute, remix, adapt, build upon this work non-commercially, and license their derivative works on different terms, provided the original work is properly cited, appropriate credit is given, any changes made indicated, and the use is non-commercial. See: http://creativecommons.org/licenses/by-nc/4.0/.

\section{REFERENCES}

1. Carroll LJ, Hogg-Johnson S, van der Velde G, et al. Course and prognostic factors for neck pain in the general population: results of the Bone and Joint Decade 2000-2010 Task Force on Neck Pain and Its Associated Disorders. Spine 2008;33:S75-S82.

2. Fejer R, Kyvik KO, Hartvigsen J. The prevalence of neck pain in the world population: a systematic critical review of the literature. Eur Spine J 2006;15:834-48.

3. Andersen LL, Mortensen OS, Hansen JV, et al. A prospective cohort study on severe pain as a risk factor for long-term sickness absence in blue- and white-collar workers. Occup Environ Med 2011;68:590-2.

4. Murray CJ, Atkinson C, Bhalla K, et al. The state of US health, 1990-2010: burden of diseases, injuries, and risk factors. JAMA 2013;310:591-608.

5. Blanpied PR, Gross AR, Elliott JM, et al. Neck pain: revision 2017. J Orthop Sports Phys Ther 2017;47:A1-A83.

6. Luime JJ, Koes BW, Miedem HS, et al. High incidence and recurrence of shoulder and neck pain in nursing home employees was demonstrated during a 2-year follow-up. J Clin Epidemiol 2005;58:407-13.

7. Borghouts JA, Koes BW, Bouter LM. The clinical course and prognostic factors of non-specific neck pain: a systematic review. Pain 1998;77:1-13.

8. Hoving JL, Gross AR, Gasner D, et al. A critical appraisal of review articles on the effectiveness of conservative treatment for neck pain. Spine 2001;26:196-205.

9. Guzman J, Hurwitz EL, Carroll LJ, et al. A new conceptual model of neck pain: linking onset, course, and care: the Bone and Joint Decade 2000-2010 Task Force on Neck Pain and Its Associated Disorders. J Manipulative Physiol Ther 2009;32(2 Suppl):S17-28.

10. Côté $P$, Wong JJ, Sutton $D$, et al. Management of neck pain and associated disorders: A clinical practice guideline from the Ontario Protocol for Traffic Injury Management (OPTIMa) Collaboration. Eur Spine J 2016;25:2000-22.

11. Bier JD, Scholten-Peeters WGM, Staal JB, et al. Clinical Practice Guideline for Physical Therapy Assessment and Treatment in Patients With Nonspecific Neck Pain. Phys Ther 2018;98:162-71.

12. Gross AR, Paquin JP, Dupont G, et al. Exercises for mechanical neck disorders: A Cochrane review update. Man Ther 2016;24:25-45.

13. Chen X, Coombes BK, Sjøgaard G, et al. Workplace-Based Interventions for Neck Pain in Office Workers: Systematic Review and Meta-Analysis. Phys Ther 2018;98:40-62.

14. Kraemer WJ, Ratamess NA, Flanagan SD, et al. Understanding the Science of Resistance Training: An Evolutionary Perspective. Sports Med 2017:47:2415-35.

15. Bird SP, Tarpenning KM, Marino FE. Designing resistance training programmes to enhance muscular fitness: a review of the acute programme variables. Sports Med 2005;35:841-51.

16. Pescatello LS, ACoS M. ACSM's guidelines for exercise testing and prescription: Wolters Kluwer/Lippincott Williams \& Wilkins Health, 2014.

17. American College of Sports Medicine. American College of Sports Medicine position stand. Progression models in resistance training for healthy adults. Med Sci Sports Exerc 2009;41:687-708.

18. Gupta S, Sharma S, Kataria C. Craniocervical Flexors Endurance Training: Treatment Approach for Cervical Spondylosis. Indian Journal of Physiotherapy and Occupational Therapy 2010;4:76-81.

19. Falla D, Lindstrøm $R$, Rechter L, et al. Effectiveness of an 8-week exercise programme on pain and specificity of neck muscle activity in patients with chronic neck pain: a randomized controlled study. Eur J Pain 2013;17:1517-28.

20. Lee MH, Park SJ, Kim JS. Effects of neck exercise on high-school students' neck-shoulder posture. J Phys Ther Sci 2013;25:571-4.

21. Andersen LL, Kjaer M, Søgaard K, et al. Effect of two contrasting types of physical exercise on chronic neck muscle pain. Arthritis Rheum 2008;59:84-91.

22. Andersen $\mathrm{CH}$, Andersen LL, Gram B, et al. Influence of frequency and duration of strength training for effective management of neck and shoulder pain: a randomised controlled trial. Br J Sports Med 2012;46:1004-10.

23. Dellve L, Ahlstrom L, Jonsson A, et al. Myofeedback training and intensive muscular strength training to decrease pain and improve work ability among female workers on long-term sick leave with neck pain: a randomized controlled trial. Int Arch Occup Environ Health 2011;84:335-46.

24. Andersen LL, Jørgensen MB, Blangsted AK, et al. A randomized controlled intervention trial to relieve and prevent neck/shoulder pain Med Sci Sports Exerc 2008;40:983-90.

25. Andersen LL, Saervoll CA, Mortensen OS, et al. Effectiveness of small daily amounts of progressive resistance training for frequent neck/shoulder pain: randomised controlled trial. Pain 2011:152:440-6.

26. Andersen $\mathrm{CH}$, Andersen LL, Pedersen MT, et al. Dose-response of strengthening exercise for treatment of severe neck pain in women. $J$ Strength Cond Res 2013;27:3322-8.

27. Rolving N, Christiansen DH, Andersen LL, et al. Effect of strength training in addition to general exercise in the rehabilitation of patients with non-specific neck pain. A randomized clinical trial. Eur J Phys Rehabil Med 2014;50:617-26.

28. Zebis MK, Andersen $\mathrm{CH}$, Sundstrup E, et al. Time-wise change in neck pain in response to rehabilitation with specific resistance training: implications for exercise prescription. PLoS One 2014;9:e93867.

29. Ylinen J, Häkkinen $A$, Nykänen $M$, et al. Neck muscle training in the treatment of chronic neck pain: a three-year follow-up study. Eura Medicophys 2007;43:161-9.

30. Ahlgren C, Waling K, Kadi F, et al. Effects on physical performance and pain from three dynamic training programs for women with work-related trapezius myalgia. J Rehabil Med 2001;33:162-9.

31. Jull GA, Falla D, Vicenzino B, et al. The effect of therapeutic exercise on activation of the deep cervical flexor muscles in people with chronic neck pain. Man Ther 2009;14:696-701.

32. Falla D, Hodges PW. Individualized Exercise Interventions for Spinal Pain. Exerc Sport Sci Rev 2017;45:105-15.

33. Jette AM. The Importance of Dose of a Rehabilitation Intervention. Phys Ther 2017;97:1043.

34. Callaghan MJ. Exercise is effective for patellofemoral pain, but what type, who benefits most and by how much remain unknown. $\mathrm{Br} J$ Sports Med 2018;52:625-6.

35. Chartered Society of Physiotherapy. James Lind Alliance Physiotherapy Priority Setting Partnership. 2018 http://www.csp.org. uk/professional-union/research/james-lind-alliance-physiotherapypriority-setting-partnership (7th Mar 2018).

36. Shamseer L, Moher D, Clarke M, et al. Preferred reporting items for systematic review and meta-analysis protocols (PRISMA-P) 2015: elaboration and explanation. BMJ 2015;350:g7647.

37. Higgins J, Green S. Cochrane Handbook for Systematic Reviews of Interventions: The Cochrane Collaboration, 2011.

38. Centre for reviews and dissemination. Systematic Reviews: CRD's Guidance for Undertaking Reviews in Health Care. University of York: York, 2009.

39. EndNote X8 [program] X8.2 version. 2018.

40. Moher D, Liberati A, Tetzlaff J, et al. Preferred reporting items for systematic reviews and meta-analyses: the PRISMA statement. BMJ 2009;339:b2535.

41. Orwin R. Evaluating coding decisions. In: Cooper H, Hedges L, eds. The handbook of research synthesis. New York: Russell Sage Foundation, 1994.

42. Cochrane Training. Data collection form for intervention reviews: RCTs and non-RCTs. 2014 http://training.cochrane.org/resource/ data-collection-forms-intervention-reviews (31st May 2018).

43. Hoffmann TC, Glasziou PP, Boutron I, et al. Better reporting of interventions: template for intervention description and replication (TIDieR) checklist and guide. BMJ 2014;348:g1687.

44. Spencer S, Wolf A, Rushton A. Spinal-exercise prescription in sport: classifying physical training and rehabilitation by intention and outcome. J Athl Train 2016;51:613-28.

45. Hozo SP, Djulbegovic B, Hozo I. Estimating the mean and variance from the median, range, and the size of a sample. BMC Med Res Methodol 2005;5:13. 
46. Gagnier JJ, Morgenstern H, Altman DG, et al. Consensus-based recommendations for investigating clinical heterogeneity in systematic reviews. BMC Med Res Methodol 2013;13:106.

47. Higgins J, Green S. Cochrane handbook for systematic reviews of intervenions: The Cochrane Collaboration, 2011.

48. Popay J, Roberts H, Sowden A, et al. Guidance on the conduct of narrative synthesis in systematic reviews: a product from the ESRC Methods Programme. Lancaster, UK: Lancaster University, 2006.

49. Ryan R. Cochrane Consumers and Communication Review Group: data synthesis and analysis: Cochrane Consumers and
Communication Review Group. 2013 http://cccrg.cochrane.org (accessed 14th May 2018).

50. Guyatt G, Oxman AD, Akl EA, et al. GRADE guidelines: 1. Introduction-GRADE evidence profiles and summary of findings tables. J Clin Epidemiol 2011;64:383-94.

51. Egger M, Davey Smith G, Schneider M, et al. Bias in meta-analysis detected by a simple, graphical test. BMJ 1997;315:629-34.

52. Dwan K, Gamble C, Kolamunnage-Dona R, et al. Assessing the potential for outcome reporting bias in a review: a tutorial. Trials 2010;11:52. 